\title{
Increased Incidence of Eosinophilia in Severe H1N1 Pneumonia during 2015 Influenza Season
}

\author{
Benjamin Deaton MD \\ Nicholas Villalobos MD \\ Andrea Mytinger DO \\ Michel Boivin MD
}

\author{
Department of Internal Medicine \\ University of New Mexico School of Medicine \\ Albuquerque, NM USA
}

\begin{abstract}
Background: A portion of patients with influenza develop a severe, life t-threatening illness requiring intensive care. We observed a significant number of critically ill influenza patients with eosinophilia during the 2015 influenza season in New Mexico. Methods: Patients were identified sequentially by reviewing disposition records of all patients admitted to the University of New Mexico Hospital medical intensive care unit between October 2015 and May 2016 for a diagnosis of influenza.

Results: Eleven patients were identified who developed respiratory failure from influenza. Average age was $43.7 \pm 11.3$ (SD) with an average SAPS-2 score of $52.0 \pm$ 13.9 (SD) on admission. All 11 were found to have H1N1 influenza. All 11 required mechanical ventilation vasopressor support. Ten patients survived. Notably, 6 (54.5\%) developed peripheral eosinophilia $(>300 / \mu \mathrm{L})$ during their hospitalization and all but one of these did not have peripheral eosinophilia at the time of admission. Bronchoalveolar lavage was performed in 5 patients $(45.5 \%)$ and none were consistent with eosinophilic pneumonia. Further data analysis revealed exploration revealed no significant differences in multiple parameters and no clear cut cause of drug-induced eosinophilia was identified.

Conclusion: During the 2015 influenza season in New Mexico, a disproportionate number of patients with $\mathrm{H} 1 \mathrm{~N} 1$ influenza and respiratory failure developed peripheral eosinophilia. Type 2 errors could have occurred due to low sample size. Given the unusual frequency of peripheral eosinophilia further studies regarding the association of influenza A and peripheral eosinophilia is warranted.

\section{Introduction}

Influenza pneumonia remains a cause of significant morbidity and mortality (1). The reemergence of H1N1 influenza in 2009 was associated with particularly severe respiratory illness, acute respiratory distress syndrome (ARDS) and mortality (2). The ARDS associated with H1N1 influenza appeared to disproportionately affect younger individuals, compared to other strains of influenza A (2). During the 2015 influenza season H1N1 circulated relatively late in the southwestern United States (3). Intensivists caring for patients with severe H1N1 pneumonia at the University of New Mexico hospital noticed a series of cases associated with significant peripheral eosinophilia.
\end{abstract}


Eosinophilia with influenza or its treatments has rarely been described (4). We therefore sought to examine all cases of severe influenza pneumonia during the 2015 influenza season for the prevalence of peripheral eosinophilia and to assess for potential associations.

\section{Methods}

This study was reviewed and approved by the Institutional Review Board of the University of New Mexico Health Sciences Center. Patients from the University of New Mexico Hospital (UNMH) adult Medical Intensive Care Unit (MICU) admitted between October 2015 through May 2016 were retrospectively screened for inclusion. Inclusion criteria included a diagnosis of influenza (using a PCR based assay of nasal swab), admission to the UNMH MICU and age $\geq 18$ years. Exclusion criteria included patients admitted to the MICU where influenza did not lead to significant respiratory failure.

In this retrospective cohort chart review, data was collected for demographics, clinical parameters at presentation and throughout their hospital course, and interventions received. Patients were assessed for the presence of eosinophilia at any point during their hospital course. Eosinophilia was defined as a serum eosinophil count that exceeded the upper limit of normal on a complete blood count $\left(0.3 \times 10^{3}\right.$ cells $/$ microliter). Values are reported with their standard deviation. Statistical analysis was performed using Stata 14 for Mac. The data was explored using two-sided $t$-tests, Fisher's exact and Chi-squared tests between the 2 groups with and without eosinophilia. The paper was partially presented in poster form at the 2017 American Thoracic Society International Congress in Washington, DC (5).

\section{Results}

Thirteen patients with influenza were identified. Two patients were excluded from further analysis as they did not meet the criteria of having respiratory failure, the remaining eleven were included in this study. The average age of patients in the study was 43.7 \pm 11.3 years with an average SAPS- 2 score of $52.0 \pm 13.9$ on admission. All eleven patients in the study admitted with severe influenza $A$ leading to respiratory failure during the 2015-2016 influenza season were found to be infected by the H1N1 strain of influenza. See Table 1 for further descriptors of the cohort. 
Table 1. Baseline and treatment characteristics by group.

\begin{tabular}{|l|l|l|l|l|}
\hline & Overall & Normal Eos & High Eos & p-value \\
\hline Sample Size & $11(100 \%)$ & $5(45.45 \%)$ & $6(54.54 \%)$ & \\
\hline Age & $43.7 \pm 11.3$ years & $43.0 \pm 12.0$ years & $44.3 \pm 11.8$ years & 0.8616 \\
\hline Gender & & & & \\
\hline \multicolumn{1}{|c|}{ Female } & $5(45.45 \%)$ & $2(40.00 \%)$ & $3(50.00 \%)$ & \\
\hline Male & $6(54.55 \%)$ & $3(60.00 \%)$ & $3(50.00 \%)$ & \\
\hline Ethnicity & & & & \\
\hline \multicolumn{1}{|c|}{ White } & $4(36.36 \%)$ & $3(60.00 \%)$ & $1(16.67 \%)$ & \\
\hline \multicolumn{1}{|c|}{ Hispanic } & $4(36.36 \%)$ & $1(20.00 \%)$ & $3(50.00 \%)$ & \\
\hline Native American & $3(27.27 \%)$ & $1(20.00 \%)$ & $2(33.33 \%)$ & \\
\hline SAPS-2 on Admission & $52.0 \pm 13.9$ & $46.2 \pm 15.3$ & $56.8 \pm 11.9$ & 0.2251 \\
\hline Vasopressors & $11 / 11(100 \%)$ & $5(100 \%)$ & $6(100 \%)$ & \\
\hline Mechanical ventilation & $11(100 \%)$ & $5(100 \%)$ & $6(100 \%)$ & \\
\hline ARDS & $11(100 \%)$ & $5(100 \%)$ & $6(100 \%)$ & \\
\hline Lowest PIF & $72.1 \pm 37.0$ & $83.6 \pm 53.1$ & $62.5 \pm 15.7$ & 0.4332 \\
\hline ECMO & $1(9.1 \%)$ & $0(0 \%)$ & $1(16.67 \%)$ & \\
\hline Tracheostomy & $4(36.36 \%)$ & $1(20.00 \%)$ & $3(50.00 \%)$ & \\
\hline ICU LOS (mean \pm SD) & $22.4 \pm 17.5$ days & $12.6 \pm 10.7$ days & $30.5 \pm 18.5$ days & 0.0898 \\
\hline Hospital LOS & $28.1 \pm 16.9$ days & $20.0 \pm 17.4$ days & $34.8 \pm 14.4$ days & 0.1561 \\
\hline
\end{tabular}

The peak eosinophil count of the group with normal eosinophil count was $0.1( \pm 0.1) \times 10^{3}$ cells/ $\mu$ l compared to $1.9( \pm 2.1) \times 10^{3}$ cells/ $\mu$ in the group with significant peripheral eosinophilia $(p=0.06)$. The range of eosinophilia in the group with normal eosinophil count was $0.0-0.3 \times 10^{3}$ cells $/ \mu \mathrm{l}$, and $0.5-4.8 \times 10^{3} \mathrm{cell} / \mathrm{s} / \mathrm{l}$ in the group with eosinophilia. The group with normal eosinophil count reached a "peak" count after an average of 4.6 days, and the group with an elevated eosinophil count after 17.1 days $(p<0.02)$.None of the patients who underwent bronchoscopy had a significant elevation in the bronchoalveolar lavage eosinophil count.

\section{Discussion}

During the 2015-2016 influenza season in New Mexico, critically ill patients at UNM hospital admitted with influenza pneumonia were infected with the H1N1 subtype. Over 50 percent of these patients developed peripheral eosinophilia at some point of their hospital course. Among those who underwent bronchoscopy, significant alveolar eosinophilia was not observed, suggesting against a pulmonary cause of eosinophilia, such as acute or chronic eosinophilic pneumonia. All patients were treated with oseltamivir, so an association with this treatment could not be determined. No demographic differences were noted between patients who had peripheral eosinophilia and those that did not. The patients with significant peripheral eosinophilia trended to have a longer ICU and hospital length of stay (LOS) but this did not reach statistical significance in this small cohort.

Type 2 errors (failure to detect a true difference between groups due to small numbers of subjects) could have occurred due to low sample size while exploring etiologies. Potential etiologies that could have explained the observed eosinophilia included drug effect, possibly due to oseltamivir, antibiotics, diuretics or other medications. A review of 
the literature reveals case reports of associations between eosinophilia and influenza vaccine $(6,7)$. Acute eosinophilic pneumonia has also been associated with H1N1 infection, but eosinophilia was not demonstrated on broncho-alveolar lavage in our series (8.9). Potentially this could have been a reaction to epitopes of this particular strain of H1N1 influenza. However, there have yet to be reports of eosinophilia during the 2015-2016 influenza season in the literature. Perhaps local factors could have contributed to an increased incidence of significant peripheral eosinophilia. Anecdotally, the authors do not however recall an increased incidence of eosinophilia in patients admitted for diagnoses other than H1N1. Patients were screened for other causes of viral pneumonia, and there was no clear co-infection that was associated with influenza associated eosinophilia. It was also noted the time to peak eosinophil count was much later in the elevated eosinophil group, and in most it took 14 days for the count to peak. This suggests the stimulus for the eosinophilia was ongoing for considerable time during the admission.

In conclusion, we describe an unusually high incidence of peripheral eosinophilia in patients with severe H1N1 influenza during the 2015 flu season. This eosinophilia was not associated with alveolar eosinophilia. Further observation for the recurrence of this association of H1N1 influenza A and peripheral eosinophilia is warranted during future influenza seasons.

\section{References}

1. Rotrosen ET, Neuzil KM, Influenza: a global perspective. Pediatr Clin North Am. 2017;64:911-36. [CrossRef] [PubMed]

2. Davlin SL, Blanton L, Kniss K, et al. Influenza Activity - United States, 2015-16 Season and Composition of the 2016-17 Influenza Vaccine.MMWR Morb Mortal Wkly Rep. 2016 Jun 10;65(22):567-75. [CrossRef] [PubMed]

3. Uyeki TM. Influenza. Ann Intern Med. 2017 Sep 5;167(5):ITC33-ITC48. [CrossRef] [PubMed]

4. Deaton, BR., Mytinger, AK, Ahmed, S, et al. Peripheral eosinophilia associated with 2016 H1N1 influenza. Am J Resp Crit Care. 2017;195:A5787 [Abstract],

5. Hayashi R, Shimomura N, Hosojima M, et al. A case of non-episodic angioedema with eosinophilia induced by influenza vaccine. Eur J Dermatol. 2017;27:554-5. [CrossRef] [PubMed]

6. Solak B, Dikicier BS, Kara RO, Erdem T. DRESS syndrome potentially induced by allopurinol and triggered by influenza vaccine. BMJ Case Rep. 2016 Mar 30;2016. [CrossRef] [PubMed]

7. Larrañaga JM, Marcos PJ, Pombo F, Otero-González I. Acute eosinophilic pneumonia as a complication of influenza $A(H 1 N 1)$ pulmonary infection. Sarcoidosis Vasc Diffuse Lung Dis. 2016 Mar 29;33(1):95-7. [PubMed]

8. Jeon EJ, Kim KH, Min KH. Acute eosinophilic pneumonia associated with 2009 influenza A (H1N1). Thorax. 2010;65:268-70. [CrossRef] [PubMed] 